\title{
Tratamento cirúrgico conservador para tumor de Warthin: relato de caso
}

\author{
Conservative surgical treatment for Warthin tumor: case report \\ Tratamiento quirúrgico conservador para tumor de Warthin: reporte de caso
}

\author{
Breno dos Reis FERNANDES \\ Henrique HADAD \\ Hernando Valentim ROCHA JÚNIOR \\ Gabriel Mulinari SANTOS \\ Bruno Coelho MENDES \\ Renato Machinez MACHADO \\ Roberta OKAMOTO \\ Jonathan Ribeiro da SILVA
}

Programa de Pós-Graduação em Odontologia, Faculdade de Odontologia de Araçatuba, Univ Estadual Paulista UNESP, 16015-050, Araçatuba-SP, Brasil

\section{Resumo}

O tumor de Warthin é a segunda lesão mais comum da glândula parótida, sendo raro em qualquer outra glândula salivar. Possui comportamento benigno, com crescimento lento e indolor. Neste artigo é relatado um caso clínico onde o tumor apresentou evolução de um ano, sem sintomatologia associada, gerando assimetria facial ao paciente, sendo realizado tratamento cirúrgico conservador apenas por enucleação da lesão, visando reduzir cirurgias maiores como a parotidectomias parciais ou totais. Após acompanhamento de 5 anos o paciente não apresenta sequelas do tratamento cirúrgico ou sinal de recorrência da lesão.

Descritores: Glândula Parótida; Neoplasia; Cirurgia Bucal.

\begin{abstract}
Warthin's tumor is the second most common lesion of the parotid gland, being rare in any other salivary gland. It has benign behavior, with slow and painless growth. In this article a clinical case was reported where the tumor presented evolution of one year, with no associated symptomatology, generating facial asymmetry to the patient, being carried out conservative surgical treatment only by enucleating of the lesion, aiming to reduce major surgeries such as partial or total parotidectomy. After 5 years of follow-up, the patient did not present sequel of the surgical treatment or a sign of recurrence of the lesion.
\end{abstract}

Descriptors: Parotid Gland; Neoplasm; Surgery, Oral.

\section{Resumen}

El tumor de Warthin es la segunda lesión más común de la glándula parótida, siendo raro en cualquier otra glándula salivar. El mismo tiene comportamiento benigno, con un crecimiento lento e indoloro. En este artículo se relata un caso clínico donde el tumor presentó evolución de un año, sin sintomatología asociada, generando asimetría facial al paciente, siendo realizado tratamiento quirúrgico conservador sólo por enucleación de la lesión, buscando reducir las cirugías mayores como la parotidectomías parciales o totales. Después del seguimiento de 5 años el paciente no presenta secuelas del tratamiento quirúrgico ni señal de recurrencia de la lesión.

Descriptores: Neoplasias; Glándula Parótida; Cirugía Bucal.

\section{INTRODUÇÃO}

O tumor de Warthin ou cistoadenoma papilífero linfomatoso, é a segunda lesão mais comum da glândula parótida, com prevalência de 6 à $14 \%$ dos tumores benignos desta glândula. Acreditase ter sua origem de tecido glandular heterotrópico, aprisionado nos linfonodos parotídeos. Acomete oito vezes mais os pacientes fumantes, e sua patogênese pode ser associada ao vírus Epstein-Barr. Acredita-se que a lesão reponde bem a cirurgia conservadora, com recidiva de 6 à $12 \%$, o que é questionável, devido a natureza multicêntrica da lesão ${ }^{1,2}$.

A lesão se manifesta clinicamente, como um aumento de volume de crescimento lento e indolor, consistente ou flutuante a palpação. Geralmente ocorre na sexta ou sétima década de vida. $\mathrm{O}$ mesmo ocorre em geral na cauda da parótida, ou seja, próximo ao ângulo mandibular ${ }^{2,3}$.

Este tumor pode ser bilateral em $10 \%$ dos casos, metacronológicos, e encontra-se no lobo superficial da parótida em mais de $80 \%$ dos casos. ${ }^{3}$ Pode exibir evolução rápida, após anos de comportamento insidioso, apresentando dor, paralisia do nervo facial e ulceração da pele ${ }^{1}$.

O diagnóstico desta lesão geralmente é feito por punção aspirativa por agulha fina, que possui bons resultados para esta patologia, entretanto a metaplasia escamosa, alteração inflamatória e fibrose podem levar a um diagnóstico incorreto. ${ }^{4,5}$ Uma segunda opção diagnóstica é a "core" biópsia, no qual obtêm-se um fragmento definitivo da lesão via cânula que se desloca no interior da neoplasia, permitindo a remoção de tecido neoplásico, para exame histopatológico ${ }^{6}$.

Esta patologia apresenta um potencial de recidiva baixo, o que permite que a mesma seja tratada de forma mais conservadora, através de dissecção extra capsular ou enucleação da mesma, visando evitar abordagens mais mórbidas como as parotidectomias parciais e totais ${ }^{7,8}$.

\section{CASO CLÍNICO}

Paciente do sexo feminino, 50 anos, leucodema, hipertenso controlado, compareceu no ambulatório de cirurgia bucomaxilofacial do Hospital Geral de Nova Iguaçu queixando-se de " rosto inchado". Ao exame físico apresentou aumento de volume assintomático, com evolução de 1 ano em região de ângulo mandibular esquerdo (Figura 1). Durante a palpação da lesão foi encontrada consistência flutuante, móvel e indolor. Ao exame de tomografia computadorizada em região de glândula parótida o tumor apresentou áreas císticas e bem delimitadas (Figuras 2 e 3). Para elaboração do diagnóstico definitivo foi realizada biópsia sob 
anestesia local. O exame histopatológico mostrou resultado compatível com tumor de Warthin.

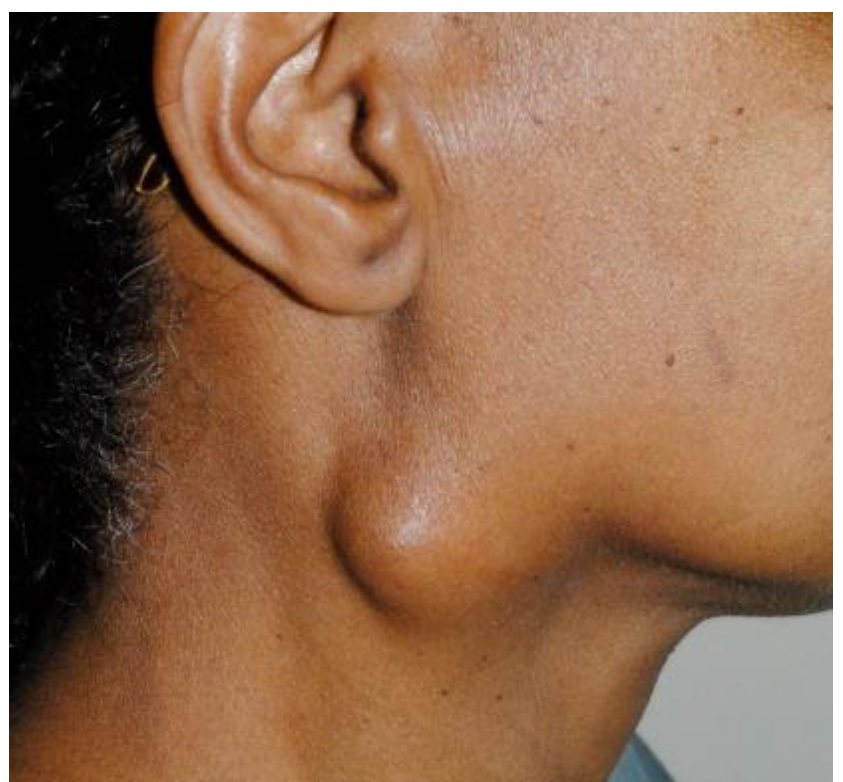

Figura 1: Aspecto clínico inicial.

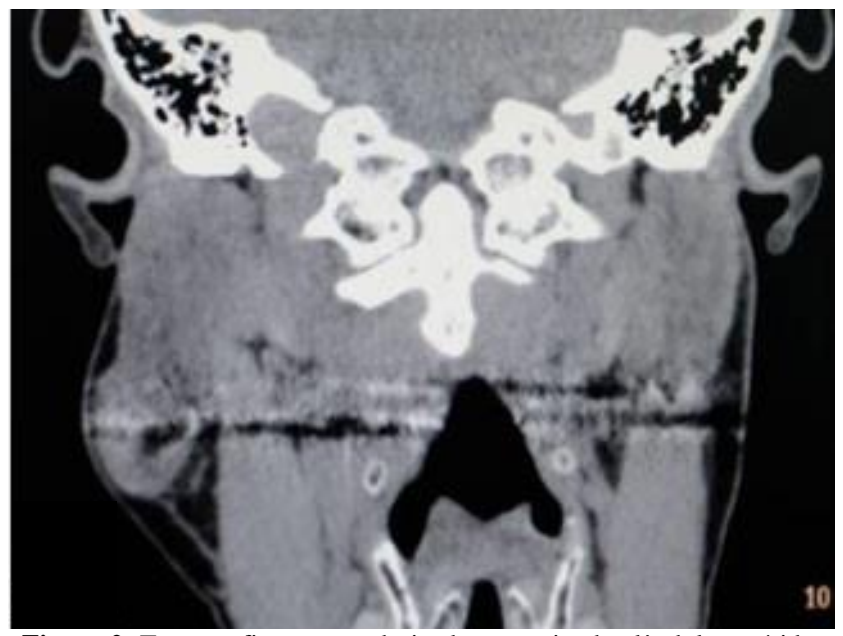

Figura 2: Tomografia computadorizada em regiãode glândula parótida.

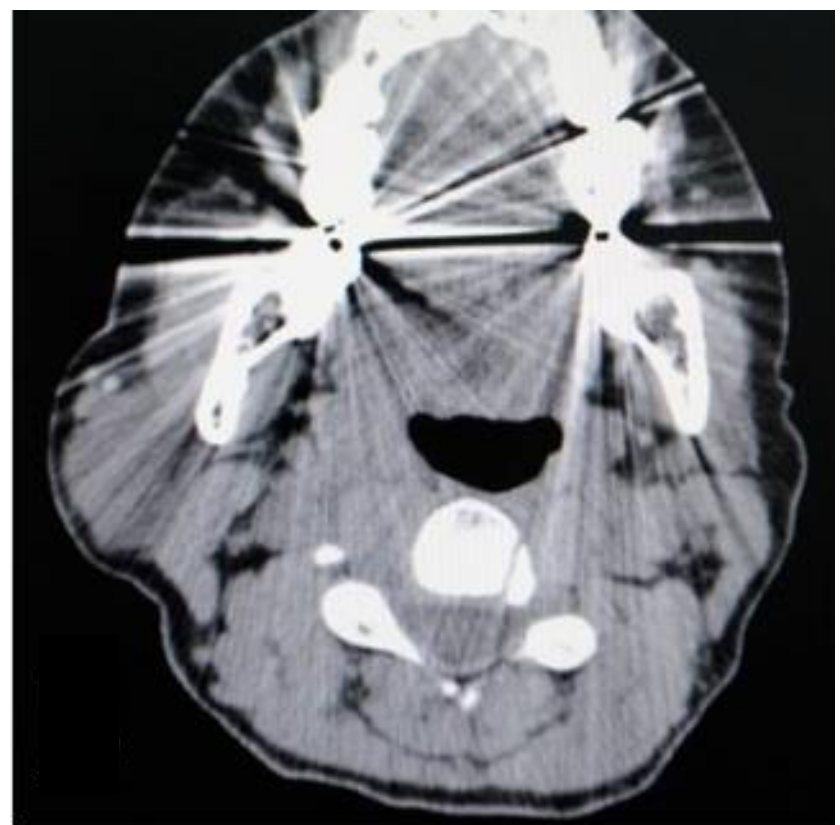

Figura 3: Tomografia computadorizada em regiãode glândula parótida.

$\mathrm{O}$ paciente foi submetido à anestesia geral com entubação nasal, sendo realizado um acesso de retromandibular para exposição da lesão que encontrava-se em intimidade com a glândula parótida (Figura 4). Após dissecção extracapsular cuidadosa, o tumor pode ser enucleado sem o rompimento da cápsula (Figura 5).

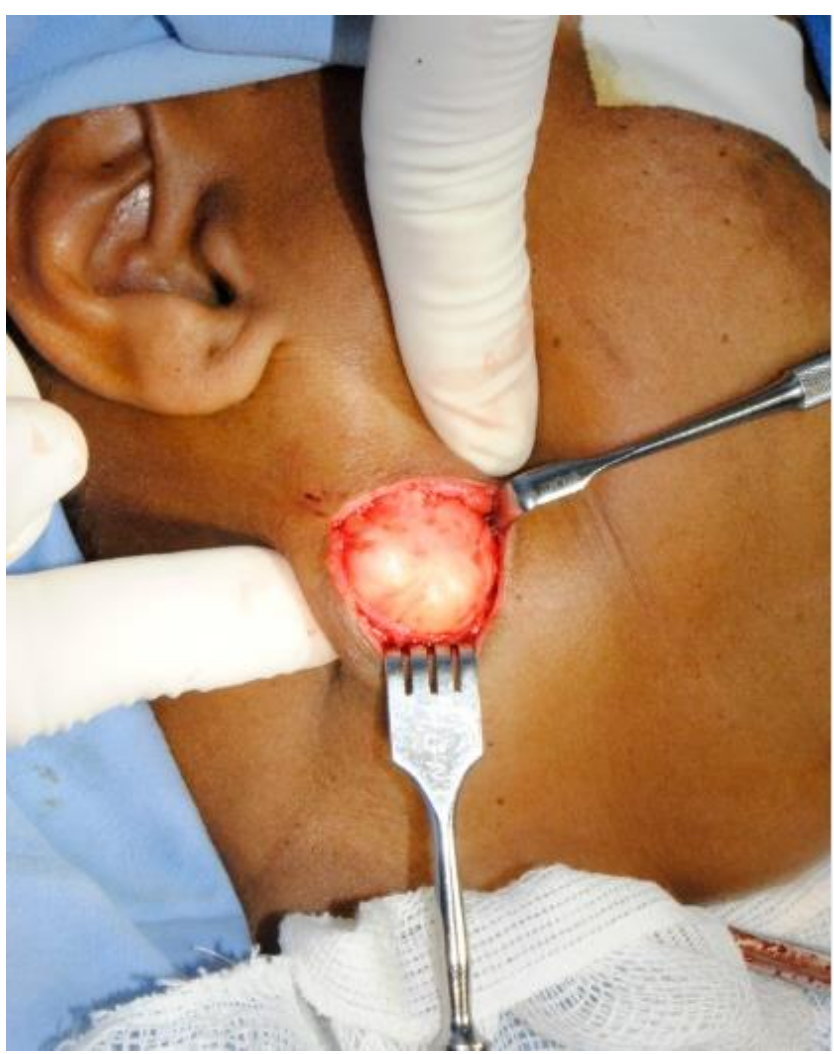

Figura 4: Aspecto trans-cirúrgico.

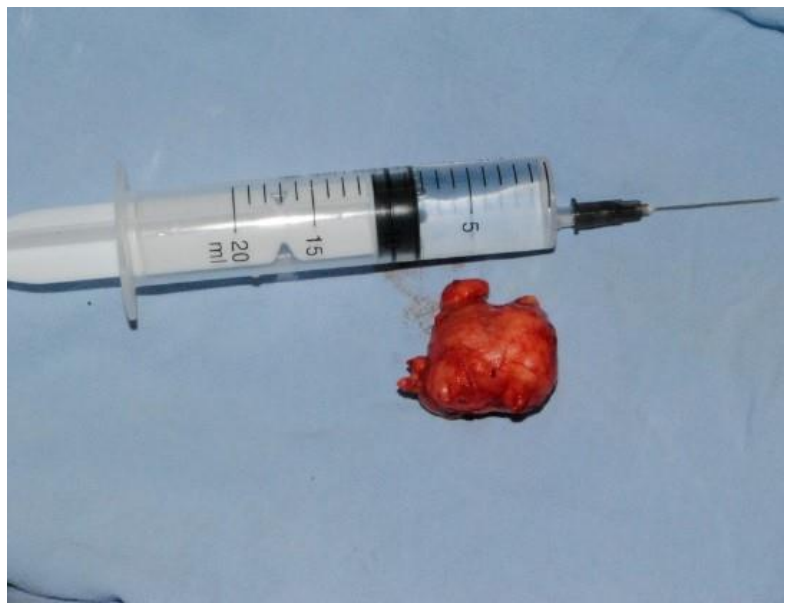

Figura 5: Aspecto macroscópico da lesão.

Os tecidos profundos foram suturados com Vycril 3.0 e a pele com Nylon 5.0, e realizou-se o curativo compressivo. No primeiro dia de pós-operatório o paciente queixou-se de dor moderada no local e apresentava pequeno edema em hemiface direita. No segundo dia após o procedimento o mesmo recebeu alta para acompanhamento ambulatorial, com prescrição de ibuprofeno $400 \mathrm{mg}$ e dipirona $500 \mathrm{mg}$ a cada 6 horas por cinco dias, além de amoxicilina $500 \mathrm{mg}$ a cada 8 horas por 07 dias. No sétimo dia de pós-operatório foi realizada a remoção de sutura, e mantido acompanhamento ambulatorial. Após 
acompanhamento de cinco anos o paciente não apresenta sequelas do tratamento cirúrgico ou sinal de recorrência da lesão.

\section{DISCUSSÃO}

O tumor de Warthin pode em raras ocasiões se apresentar bilateralmente e simultâneos, também pode estar associado à outra neoplasia de glândula salivar, sendo a combinação mais comum o tumor de Warthin associado ao adenoma pleomórfico ${ }^{9}$.

Estudos demostram que as técnicas menos invasivas como a dissecção extra capsular podem reduzir o risco de paralisia do nervo facial, síndrome de Frey e permitem uma remoção de tecido glandular sadio menor. Esta técnica, quando bem indicada não aumentou consideravelmente os índices de recidiva das lesões ${ }^{8,10}$. No presente caso onde foi realizado a enucleção com dissecção extracapsular, não houve desenvolvimento de síndrome de Frey, paralisia do nervo facial ou hematoma ${ }^{11}$.

Alguns autores afirmam que os tumores de até $4 \mathrm{~cm}$ podem ser tratados pela dissecção extracapsular, enquanto as lesões maiores deveriam ser tratadas por parotidectomia parcial. Mesmo através da parotidectomia parcial, as lesões não possuem margem de segurança, pois muitas vezes, estarão associadas diretamente ao nervo facial, sendo apenas enucleadas do nervo ${ }^{7,12}$.

Segundo Piekarski et $\mathrm{al}^{13}$ em seu estudo sobre dissecção extra capsular, o percentual de recidiva das lesões tratadas por esta técnica é de $8.2 \%$ em um acompanhamento médio de 34 meses. As lesões de 4 a $6 \mathrm{~cm}$ foram as que apresentaram menor potencial de recidiva em dez anos, devido a maior remoção do parênquima da superfície glandular. Já as lesões de 2 a $4 \mathrm{~cm}$, apresentaram maior potencial de recidiva em dez anos, causada por perfuração e rompimento da capsula.

Laskawi et al. $^{14}$ em seu estudo sobre adenomas pleomórficos observaram nenhuma recorrência das lesões tratadas por parotidectomia total, $0.7 \%$ de recorrência das lesões tratadas por parotidectomia parcial, e $7.2 \%$ de recorrência das lesões tratadas por enucleação, o que permite um encorajamento a técnica conservadora, visto que o tumor de Warthin, possui menor potencial de recidiva que $\mathrm{o}$ adenoma pleomórfico.

Ethunandan et al. ${ }^{10}$ descrevem o tumor de Warthin como uma lesão de origem multicêntrica em $12 \%$ à 50\% dos casos. Logo, muitas lesões creditadas como recorrentes, podem na realidade serem outro foco do tumor multicêntrico. Estes mesmos autores afirmam que a ruptura da cápsula da lesão não é um fator de risco para recorrência. Sugerem ainda que lesões solitárias de tumor Warthin podem ser tratadas por enucleação e dissecção extracapsular, devido ao baixo índice de recidiva.

$\mathrm{Na}$ cirurgia radical como a parotidectomia parcial, a glândula parótida muitas vezes perde a sua função, devido à impossibilidade de manutenção do ducto de Stenon, ou por complicações transoperatórias. Esta situação não se repete nas técnicas conservadoras, tal como dissecção extra capsular e enucleação ${ }^{15}$.

Alguns trabalhos mostram que o índice de síndrome de Frey foi de $17 \%$ das lesões tratadas por parotidectomia total, $14 \%$ das lesões tratadas por parotidectomia parcial e nenhum caso nas lesões tratadas por enucleação ${ }^{13}$. O uso de retalhos pediculado do músculo esternocleidomastóideo e da fáscia da glândula parótida, tem sido utilizado na tentativa de reduzir a incidência desta síndrome, com bons resultados ${ }^{16,17}$.

Ambas as técnicas citadas no texto são descritas na literatura, o que permite a utilização de todas. As cirurgias mais radicais são soberanas no tratamento das lesões como o adenoma pleomórfico, devido seu alto potencial de recidiva. Todavia, lesões como tumor de Warthin apresentam menor índice de recorrência, o que permite optar por cirurgias conservadoras. A dissecção extracapsular e a enucleação são opções extremamente válidas no tratamento do tumor Warthin com baixa recorrência. Estas técnicas também causam menor número de complicações com paralisia do nervo facial e síndrome de Frey.

O índice de recorrência da lesão pode estar diretamente associado à multicentricidade da mesma, remoção parcial da cápsula e penetração capsular. Em todas as técnicas cirúrgicas, não haverá margem total de tecido sadio, pois muitas vezes o tumor estará sobreposto ao nervo facial, sendo apenas sua cápsula enucleada deste nervo.

\section{CONSIDERAÇÕES FINAIS}

Conclui-se que, a opção cirúrgica por enucleção, é de grande valia no arsenal do cirurgião, principalmente nas lesões que estão localizadas no lobo superficial, e apresentam-se bem delimitadas e circunscritas, com pouco envolvimento de tecido glandular sadio.

\section{REFERÊNCIAS}

1. Dedivitis RA, Pfeutzenreiter Jr EG. Tumor de Warthin: revisão de 60 casos. Rev Bras Cir Cabeça Pescoço. 2010; 39(4):239-41.

2. Neville BW, Damm DD, Allen CM, Bouquot JE. Patologia Oral e Maxilofacial. 2.ed., 2004.

3. Linares LP, Urízar JMA, Aytés LB, Escoda CG. Papillary cistoadenoma lymphomatousum (Wathin-like) of minor salivary glands. Med Oral Patol Oral Cir Bucal. 2009;14(11):597-600.

4. Stárek I, Skálová A, Tichý T. Histopathologic changes in parotid gland parenchyma after fine nedle aspiration biopsy of a Warthin's tumour. A case report. Pathol Res Pract. 2002;198:829-32. 
5. Malinsky RR, Dall'igna DP, Smith MM, Costa SS. Punção aspirativa por agulha fina em tumores cervicais. Rev Bras Otorrinolaringol. 2002; 68(3):395-98.

6. Wan YL, Chan SC, Chen YL, Cheung YC, Lui $\mathrm{KW}$, Wong HF et al. Ultrasonography-guided core needle biopsy of parotid gland masses. Am J Neuroradiol. 2004; 25(9):1608-12.

7. Emodi O, El-naaj IA, Gordin A, Akrisb S, Peled M. Superficial parotidectomy versus retrograde partial parotidectomy in treating benign salivary gland tumor (pleomorphic adenoma). J Oral Maxillofac Surg. 2010; 68(9):2092-98.

8. Shehata EA. Extra-capsular dissection for benign parotid tumours. Int J Oral Maxillofac Surg. 2010; 39(2):140-44.

9. Toida M, Mukai K, Shimosato Y, Ebihara S. Simultaneous occurrence of bilateral Warthin's tumors and pleomorphic adenoma in the parotid glands. J Oral Maxillofac Surg. 1990; 48(10):1109-13.

10. Ethunandam M, Pratt CA, Higgins B, Morrison A, Umar T, Marcpherson DW et al. Factors influencing the occurrence of multicentric and 'reccurent' Warthin's tumour: a cross sectional study. Int J Oral Maxillofac Surg. 2008; 37(9):831-34.

11. Gomes-Ferreira PH, de Carvalho Reis EN, Faverani LP, de Oliveira D, Carrasco LC, Vieira JM. Frey syndrome after trauma: diagnosis and treatment. J Craniofac Surg. 2017; 28(2):582-83.

12. IizucaI K, Ishikawa K. Surgical techniques for benign parotid tumors: segmental resection vs extracapsular lumpectomy. Acta Otolaryngol. $1998 ; 537: 75-81$.

13. PiekarskiI J, Nejc D, Szymczak W, Wronski K., Jeziorski A. Results of extracapsular dissection pleomorphic adenoma of parotid gland. J Oral Maxillofac Surg. 2004; 62(10):1198-1202.

14. Laska WIR, Schott T, Mirzaie-Petri M, Schroeder M. Surgical management of pleomrophic adenomas of the parotid gland: a follow-up study of three methods. J Oral Maxillofac Surg. 1996; 54(10):1176-79.

15. Kun Z, Dao-Yi Q, Li-Min W. Functional superficial parotidectomy. J Oral Maxillofac Surg. 1994; 52(10):1038-41.

16. Santos RC, Chagas JFS, Bezerra TFP, Baptistella JE. Pagani MA, Melo AR. Prevalência da Síndrome de Frey após parotidectomia parcial. Rev Bras Otorrinolaringol. 2006; 72(1):112-15.

17. Nofal AAF, Mohamed M. Sternocleidomastoid muscle flap after parotidectomy. Int Arch Otorhinolaryngol. 2015; 19(4):319-24.

\section{CONFLITO DE INTERESSES}

Os autores declaram não haver conflitos de interesse.

AUTOR PARA CORRESPONDENCIA

Breno dos Reis Fernandes

brenofernandesctbmf@hotmail.com

Submetido em 10/04/2018

Aceito em 06/06/2018 